\title{
Aluminum Hydroxide Nanosheets with Structure-dependent Storage and Transportation toward Cancer Chemotherapy
}

\author{
LI Xia ${ }^{1}$, SHENASHEN Mohamed A ${ }^{1}$, MEKAWY Moataz ${ }^{1}$, TANIGUCHI Akiyoshi ${ }^{2,3}$, EI-SAFTY Sherif A ${ }^{1,3}$ \\ (1. Research Center for Functional Materials, National Institute for Materials Science, Ibaraki 305-0047, Japan; 2. Cellular \\ Functional Nanomaterials Group, Research Center for Functional Materials, National Institute for Materials Science, Ibaraki \\ 305-0044, Japan; 3. Engineering and Advanced Manufacturing, University of Sunderland, Sunderland, United Kingdom)
}

\begin{abstract}
Alum has an excellent safety record and is the only licensed inorganic adjuvant for human vaccines. However, the exploration of alum nanosheets as chemotherapy drug delivery system, especially the clarification about the relationship between structures and drug loading properties, is totally insufficient. Herein, aluminum hydroxides $(\mathrm{AlOOH})$ nanosheets with tunable specific surface area and pore size were synthesized by adjusting the synthesis time in the presence of triblock copolymers. The obtained materials exhibited the highest surface area about $470 \mathrm{~m}^{2} / \mathrm{g}$. The structure-dependent chemotherapy drug loading capability for $\mathrm{AlOOH}$ nanosheets was observed: the higher specific surface area and pore size are, the higher amount of chemotherapy drug is loaded. AlOOH nanosheets loaded with doxorubicin showed a $\mathrm{pH}$-dependent sustained release behavior with quick release in low $\mathrm{pH}$ about 5 and slow release in $\mathrm{pH}$ around 7.4. Doxorubicin-loaded $\mathrm{AlOOH}$ nanosheets exhibited much higher cancer cellular uptake efficiency than that in free form by flow cytometry. Moreover, doxorubicin-loaded AlOOH nanosheets with high specific surface area showed an increased cellular uptake efficiency and enhanced ratios of apoptosis and necrosis, compared with those showing low specific surface area. Therefore, $\mathrm{AlOOH}$ nanosheets are promising materials as chemotherapy drug delivery system.
\end{abstract}

Key words: aluminum hydroxide; nanosheet; cancer chemotherapy; storage; drug delivery

The World Health Organization reported that approximately 8.2 million people worldwide die from cancer annually, accounting for an estimated $13 \%$ of all deaths. Chemotherapy is a major type of cancer treatment that involves the use of cytotoxic chemotherapeutic agents to treat cancer cells that divide rapidly. However, chemotherapy through systematic administration suffers from severe side effects on normal tissues ${ }^{[1]}$ and from multiple drug resistance $(\mathrm{MDR})^{[2-3]}$, which is responsible for the active expulsion of chemotherapy drugs from tumor cells and the survival of tumor cells treated with the drugs; the mechanism underlying this phenomenon involves the overexpression of P-glycoprotein, MDRassociated protein, and so on. Nanocarriers that encapsulate toxic drugs can minimize damage to normal organs and decrease the MDR of cancer cells ${ }^{[4-6]}$. An ideal drug carrier should possess excellent safety properties, accommodate large amounts of drug molecules, diffuse effectively through cancer cells, display controlled re- lease behavior, and so on.

Two-dimensional (2D) nanosheets (NSs), such as graphene, silicate, transition metal dichalcogenides, and transition metal oxides have attracted tremendous interest as a newly emerging class of nanomaterials in drug delivery systems. These NSs possess a unique planar topography, ultrathin thickness, high degree of anisotropy, high surface-area-to-mass ratio, and the ability to adsorb large amounts of drug molecules ${ }^{[7-9]}$. Thus far, no study has reported the synthesis of aluminum hydroxide NSs with a tailored surface area and explored their use as chemotherapy drug carriers. Alum in the form of aluminum hydroxide, aluminum phosphate, or aluminum sulfate has been used in human vaccines for nearly a hundred years because of its lack of side effects; however, its mechanism remains unclear ${ }^{[10-13]}$. Alum is the only licensed inorganic adjuvant for human use by the Food and Drug Administration of the United States ${ }^{[10-12]}$. To date, few reports focused on tuning the shape, crystallin-

Received date: 2019-01-25; Revised date: 2019-08-06

Foundation item: National Institute for Materials Science of Japan

Biography: LI Xia (1980-), female, PhD. E-mail: lixia6969@hotmail.com

李 霞(1980-), 女, 博士. E-mail: lixia6969@hotmail.com

Corresponding author: EI-SAFTY Sherif A. E-mail: Sherif.ELSAFETY@nims.go.jp 
ity, and surface reactivity of alum and on its effects on the biological system (such as the triggering NLRP3 inflammasome activation in THP-1 cells and murine bone marrow-derived dendritic cells $)^{[14]}$. Precise control over aluminum hydroxide $(\mathrm{AlOOH}) \mathrm{NSs}$ with tailored structures may exert a distinct influence on the accessibility of drug molecules, the interaction between drug molecules and the carriers, and the biological responses. Previous findings indicate that $\mathrm{AlOOH}$ NSs may serve as promising inorganic carriers with the lowest barrier for clinical transfer, but still need clarification about the relationship between structures and drug loading properties.

The present study reports a simple one-pot fabrication of AlOOH NSs with a tunable surface area and pore size by using P123 templates (scheme 1). Then, we establish the correlation between the nanostructure and chemotherapy drug loading capability of the NSs. To the best of our knowledge, this study is the first to report the effects of surface area and pore size of AlOOH NSs on chemotherapy drug delivery properties. Moreover, the cellular uptake and cancer cell viability of $\mathrm{AlOOH}$ NSs loaded with doxorubicin (Dox) were studied using DU145 prostate cancer cells. The NSs also showed good biocompatibility in vivo.

\section{Experimental}

\subsection{Synthesis of aluminum oxyhydroxide nanosheets}

Aluminum oxyhydroxide nanosheets were synthesized by using the inorganic aluminum salt $\mathrm{AlCl}_{3}$ as $\mathrm{Al}$ source, urea and triblock copolymer P123 as templates. In a typical synthesis, $0.00016 \mathrm{~mol} \mathrm{P123}$ and $0.01 \mathrm{~mol}$ $\mathrm{AlCl}_{3} \cdot 6 \mathrm{H}_{2} \mathrm{O}$ were mixed in $20 \mathrm{~mL}$ water and aged in a shaking bath at $40{ }^{\circ} \mathrm{C}$ for 1 . Then, 0.04 mol urea was added to the above sol solution, which was hydrother- mally treated at $100{ }^{\circ} \mathrm{C}$ for 6.5 and $24 \mathrm{~h}$, respectively. The final products were collected by centrifugation, extracted in ethanol for $1 \mathrm{~d}$ to remove the templates, washed with water and ethanol for 3 times, and named $\mathrm{Al}-\mathrm{L}$ and $\mathrm{Al}-\mathrm{H}$, respectively.

\subsection{Characterization}

The morphology and structure of the nanosheets were observed under a JEM-2100 transmission electron microscope. Wide-angle powder X-ray diffraction (XRD) patterns were obtained using an $18 \mathrm{~kW}$ diffractometer (Bruker D8 Advance) with monochromated $\mathrm{Cu} \mathrm{K} \alpha$ radiation. Zeta potential was analyzed using a zeta potential and particle size analyzer (ELSZ-1000, Photal, Otsuka Electronics) in an appropriate buffer. An ultravioletvisible-near infrared spectrophotometer (SolidSpec-3700, Shimadzu) was used to record the Dox concentration before and after loading. The release curve of Dox from the materials was measured using a microplate reader with absorbance at $490 \mathrm{~nm}$. The nitrogen adsorptiondesorption isotherms were recorded on a BELSORP36 analyzer at $77 \mathrm{~K}$ under a continuous adsorption condition. Brunauer-Emmett-Teller and Barrett-Joyner-Halenda analyses were conducted to determine the surface area, pore size, and pore volume.

\subsection{Drug loading and release}

A $100 \mu \mathrm{g}$ portion Al-L or Al-H mesoporous materials in $500 \mu \mathrm{L}$ phosphate-buffered saline (PBS) solution ( $\mathrm{pH}$ 8.0) and $50 \mu \mathrm{L}$ Dox solution at $1 \mathrm{mg} / \mathrm{mL}$ in water were mixed and shaken at $100 \mathrm{r} / \mathrm{min}$ overnight at room temperature. Then, the mixture was centrifuged and washed once to obtain the final Al-L/Dox or Al-H/Dox complexes.

\subsection{Apoptosis/necrosis assay and cellular up- take assay by flow cytometry}

DU145 prostate cancerous cells with a density of approximately $4 \times 10^{4}$ cells $/ \mathrm{cm}^{2}$ were cultured in $35 \mathrm{~mm}$

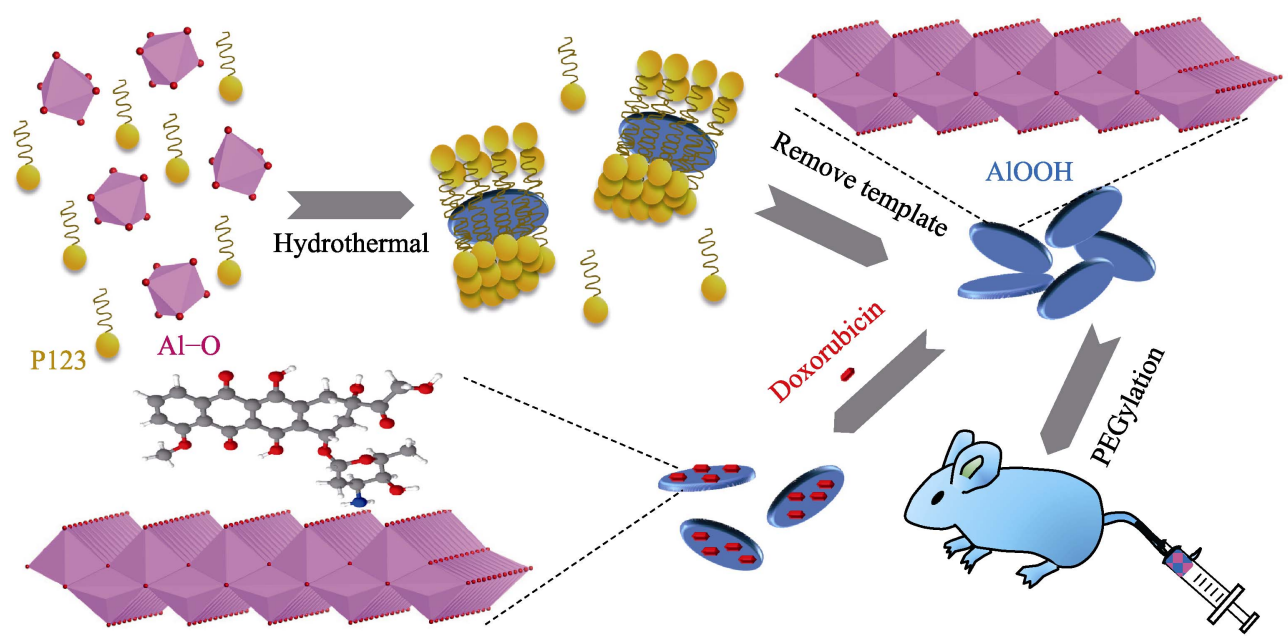

Scheme 1 Formation of AlOOH NSs, loading of chemotherapy drugs onto NSs, and intravenous injection of the as-prepared NSs 
dishes containing RPMI1640 medium supplemented with $10 \%$ fetal bovine serum at $37{ }^{\circ} \mathrm{C}$ in humidified air containing $5 \% \mathrm{CO}_{2}$ overnight. Al-L or Al-H suspensions loaded with Dox were added into the above dishes at a final concentration of 5 and $25 \mu \mathrm{g} / \mathrm{mL}$, respectively. Free Dox at an equivalent amount as that released from Al-H/Dox after $1 \mathrm{~d}$ at $\mathrm{pH} 5$ were used as contrast. After $3 \mathrm{~h}$ of incubation, the cellular uptake efficiency of Dox was recorded with a flow cytometry analyzer (ICyt EC800). Moreover, after $1 \mathrm{~d}$ of incubation, apoptotic and necrotic cells were double-stained with Annexin V-FITC and propidium iodide $(\mathrm{PI})$, and recorded by using the flow cytometry analyzer.

\subsection{Direct observation of cellular uptake by confocal laser scanning microscope}

For investigating the cellular uptake of Al-H/Dox complexes, fluorescein isothiocyanate (FITC) was conjugated with Al-H with the aid of 3-aminopropyltriethoxysilane to obtain FITC-Al-H samples. Then, Dox was loaded onto the FITC-Al-H samples under the same conditions mentioned above. DU145 cancer cells were seeded in a $35 \mathrm{~mm}$ glass bottom Petri dish at a density of $4 \times 10^{4}$ cells $/ \mathrm{cm}^{2}$. After incubation of cells overnight, FITC-Al-H/Dox complexes were added in the dish at a final concentration of $25 \mu \mathrm{g} / \mathrm{mL}$. After $0.5,2.5$ and $24 \mathrm{~h}$ of incubation, the cells were washed twice with PBS and fixed with $4 \%$ paraformaldehyde for $20 \mathrm{~min}$. Afterward, the cell nucleus was stained with Hoechst. The cellular uptake of the complexes was visualized under a confocal laser scanning microscope (CLSM, SP5, Leica).

\subsection{In vivo safety test}

To examine the in vivo safety, C57/BL6 mice were administered intravenously via tail vein injection of Al-H (50 $\mu \mathrm{g}$ in PEG/saline), and acute toxicology, such as blood biochemistry, was measured after $3 \mathrm{~d}$. All animal experiments were approved by the Ethical Committee of the National Institute for Materials Science (NIMS), Japan. All animal experiments and feeding were carried out in accordance with the guidelines of the Ethical Committee of NIMS, Japan.

\section{Results and discussion}

AlOOH NSs with a tunable specific surface area and pore size were hydrothermally synthesized by using the inorganic aluminum salt $\mathrm{AlCl}_{3}$ as $\mathrm{Al}$ source, urea as alkaline source and triblock copolymer P123 as templates. The hydrothermal products obtained at $100{ }^{\circ} \mathrm{C}$ for 6.5 and $24 \mathrm{~h}$ were named Al-L and Al-H, respectively. TEM images of Al-L and Al-H show the morphology of NSs with particle size of 10-30 nm (Fig. 1(A)). AFM image of Al-H show the thickness of NSs around 2 nm (Fig. 1(B)). The nitrogen adsorption-desorption isotherms (Fig. 1(C)) of Al-L and Al-H show a capillary condensation step in the relative pressure ranges of $0.4-0.6$ and $0.45-0.8$, respectively. Fig. 1(C) inset shows that the mesopore sizes of Al-L and Al-H center at approximately 3 and $5 \mathrm{~nm}$, respectively. The surface area and pore volume were calculated to be $287 \mathrm{~m}^{2} / \mathrm{g}$ and $0.24 \mathrm{~cm}^{3} / \mathrm{g}$ for Al-L, and $470 \mathrm{~m}^{2} / \mathrm{g}$ and $0.53 \mathrm{~cm}^{3} / \mathrm{g}$ for Al-H, respectively. Wide-angle $\mathrm{X}$-ray diffraction patterns demonstrate the presence of broadening reflection peaks (020), (120) and (031), which corresponds to the formation of the $\gamma-\mathrm{AlOOH}$ phase (boehmite) with a low crystallinity (Fig. 1(D)). Importantly, the obtained Al-H samples exhibited an extremely high specific surface area compared with those in previous reports ${ }^{[15-18]}$. For instance, $\gamma$-AlOOH hollow microspheres prepared by a hydrothermal precipitation reaction of potassium aluminum sulfate in the presence of urea in pure water yield a BET surface area of $93.6 \mathrm{~m}^{2} / \mathrm{g}^{[15]}$. In another case, nanostructured boehmite synthesized via a solvothermal route using aluminum nitrate and an isopropanol-toluene mixture as solvent shows a specific surface area of ca. $264.7 \mathrm{~m}^{2} / \mathrm{g}^{[16]}$. In addition, curtain-like crumpled boehmite prepared by using aluminum sulfate and hexamethylenetetramine displays a specific surface area of $353 \mathrm{~m}^{2} / \mathrm{g}$, whereas theoretical calculation demonstrates that one-lattice boehmite sheets possess a surface area of $529 \mathrm{~m}^{2} / \mathrm{g}^{[18]}$. Although mesoporous boehmite by the hydrolysis reaction of aluminum sec-butoxide in an ethanol-water solution with an amine surfactant reportedly possesses a high surface area of $462 \mathrm{~m}^{2} / \mathrm{g}$, the utilization of expensive and toxic aluminum organic reagents did not facilitate its biomedical application ${ }^{[19]}$. In the present study, we use the cheap aluminum inorganic salt $\mathrm{AlCl}_{3}$ as aluminum source to obtain aluminum hydroxide with a high specific surface area and prevent the use of expensive and toxic aluminum organic reagents.

The structure-dependent chemotherapy drug loading capability and release behavior of the AlOOH NSs were verified by using the chemotherapy drugs Dox (Fig. 1(E)). The Al-H sample with a high surface area and large pore size showed about two times higher Dox loading ability compared with the Al-L sample with a low surface area and small pore size. The loading efficiency of Dox onto Al-L and Al-H is $14 \%$ and $31 \%$, respectively. The surface area-dependent drug loading ability of the AlOOH NSs was also observed in a previous mesoporous silica delivery system loaded with the anti-inflammation drug ibuprofen ${ }^{[20]}$. The storage capacity of nanocarriers highly depends on the specific surface area and pore volume of the mesoporous carrier; a larger specific surface area and 

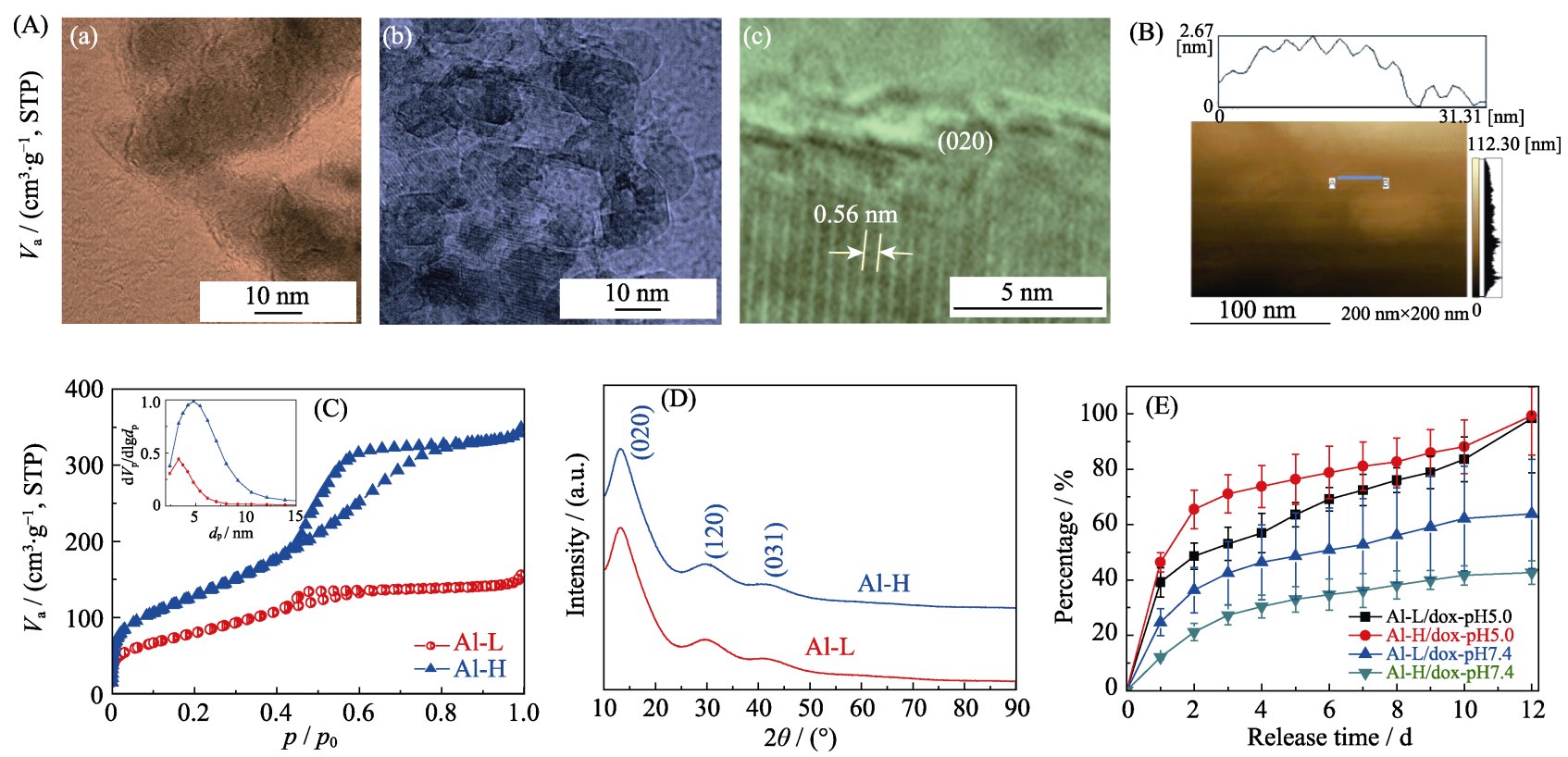

Fig. 1 (A) Transmission electron micrograph images of Al-L (a) and Al-H (b, c); (B) Atomic force microscopy image of Al-H; (C) $\mathrm{N}_{2}$ sorption isotherms and pore size distribution curves of the samples;

(D) XRD patterns of the samples; (E) The release curves of Dox for the AlOOH NSs

pore volume leads to a higher storage capacity ${ }^{[20]}$. Notably, the AlOOH NSs loaded with Dox showed a pH-dependent sustained release behavior with a high release at a low $\mathrm{pH}$ of approximate 5 but a low release at a $\mathrm{pH}$ of around 7.4. For example, the Al-H/Dox samples exhibited a high release of $(46.4 \% \pm 3.5 \%)$ at day 1 and $(99 \% \pm 14 \%)$ at day 12 at a low pH of 5 but a low release of $(12.1 \% \pm 0.9 \%)$ at day 1 and $(42.7 \% \pm 4.3 \%)$ at day 12 at $\mathrm{pH} 7.4$.

The zeta potentials of the AlOOH NSs before and after Dox loading were recorded to analyze the interaction between particles and Dox molecules. The zeta potential increased from $-21.29 \mathrm{mV}$ to $-7.23 \mathrm{mV}$ after Dox loading onto the Al-H sample because of the positive charge of Dox with amine group. Meanwhile, the zeta potential changed from $-24.71 \mathrm{mV}$ to $-16.90 \mathrm{mV}$ after Dox loading onto the Al-L sample particles. The similar zeta potential for the initial Al-H and Al-L samples and the larger zeta potential change for the Al-H samples after Dox loading are consistent with the higher Dox loading amount. In addition, the AlOOH NSs displayed the following $\mathrm{pH}$-dependent zeta potentials: $23.21 \mathrm{mV}$ at $\mathrm{pH} 5$, $-17.15 \mathrm{mV}$ at $\mathrm{pH} 7.4$, and $-21.29 \mathrm{mV}$ at $\mathrm{pH}$ 8. The negative charge at a high $\mathrm{pH}$ of around 8.0 resulted in the electrostatic attraction between the $\mathrm{AlOOH}$ NSs and Dox molecules, whereas the positive charge at a low $\mathrm{pH}$ of around 5.0 led to electrostatic repulsion between each other and the release of loaded Dox molecules.

The cellular uptake of the Al-H/Dox samples was observed under a confocal laser scanning microscope. Fluorescein isothiocyanate (FITC)-conjugated Al-H samples were used to load Dox to form FITC-Al-H/Dox complexes. The obtained complexes were incubated with DU145 cells for different times and then observed. As shown in Fig. 2(A), green fluorescence emitted by FITC-Al-H was visualized in cells and primarily distributed near the cell membrane. This result suggests that the particles loaded with Dox were endocytosed by DU145 cells and internalized into the cells. The internalized amounts of FITC-Al-H/Dox samples in the cytoplasm and the Dox amount in the nucleus evidently increased with prolonged time from $0.5 \mathrm{~h}$ to $24 \mathrm{~h}$.

The quantitative calculation of the cellular uptake of the Al-L/Dox, Al-H/Dox, and free Dox samples was conducted using flow cytometry based on the red fluorescence of Dox (Fig. 2(B, C)). At $5 \mu \mathrm{g} / \mathrm{mL}$ of the initial AlOOH NS particles, the Al-H/Dox samples exhibited a considerably higher cellular uptake percentage of $86.59 \%$ compared with the Al-L/Dox samples (26.92\%) because of the higher Dox loading ability of the Al-H samples. Although both Al-L/Dox and Al-H/Dox samples showed almost $100 \%$ of cellular uptake at higher particle concentration, the peak fluorescence intensity evidently shifted to the right side for the Al-H/Dox samples (data not shown). Moreover, the cellular uptake percentages of DU145 cells for Al-H/Dox and free Dox were 86.59\% and $41.51 \%$, respectively, when the Dox amount was normalized to that loaded onto $5 \mu \mathrm{g} / \mathrm{mL}$ of Al-H particles. Similarly, at higher concentration, the fluorescence intensity of Al-H/Dox was considerably higher than that of free Dox, regardless of their $99.24 \%$ and $97.22 \%$ cellular uptake percentages, respectively (data not shown). 

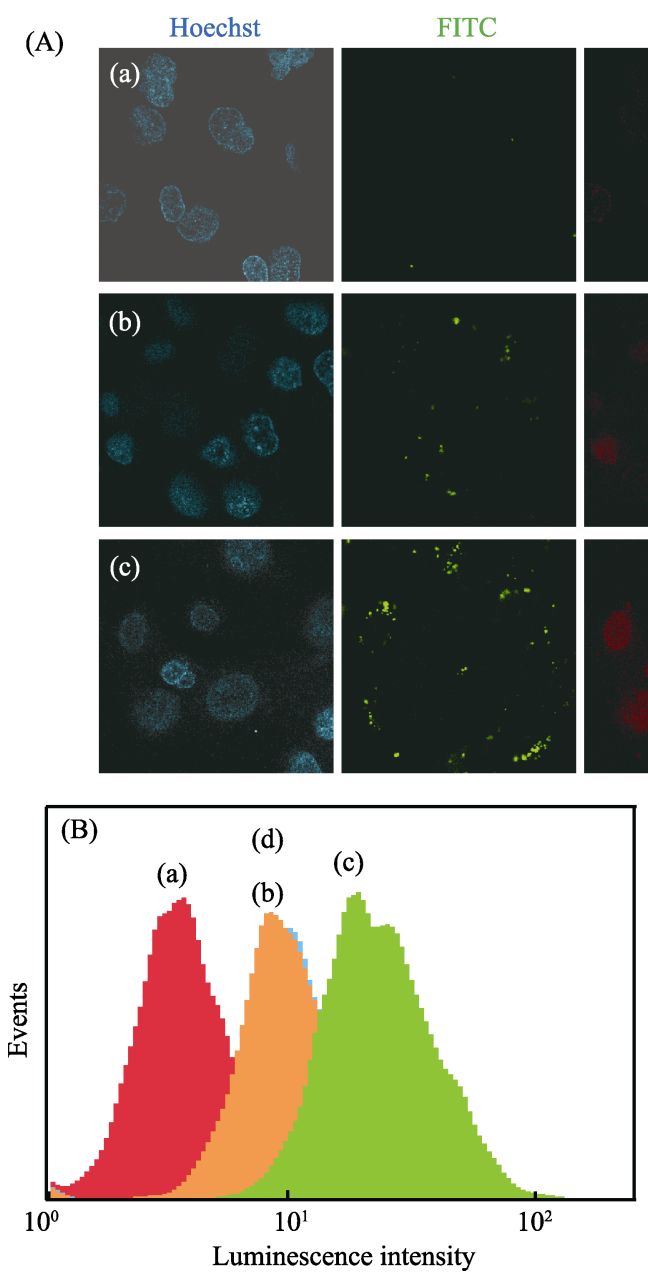

FITC
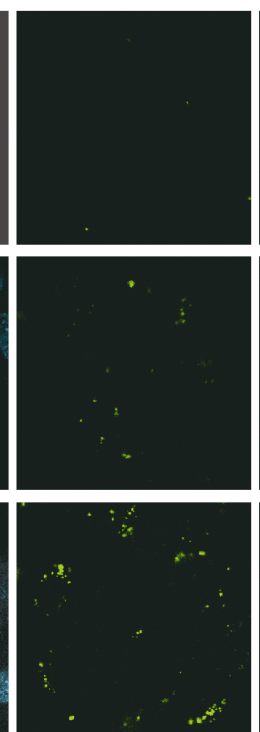
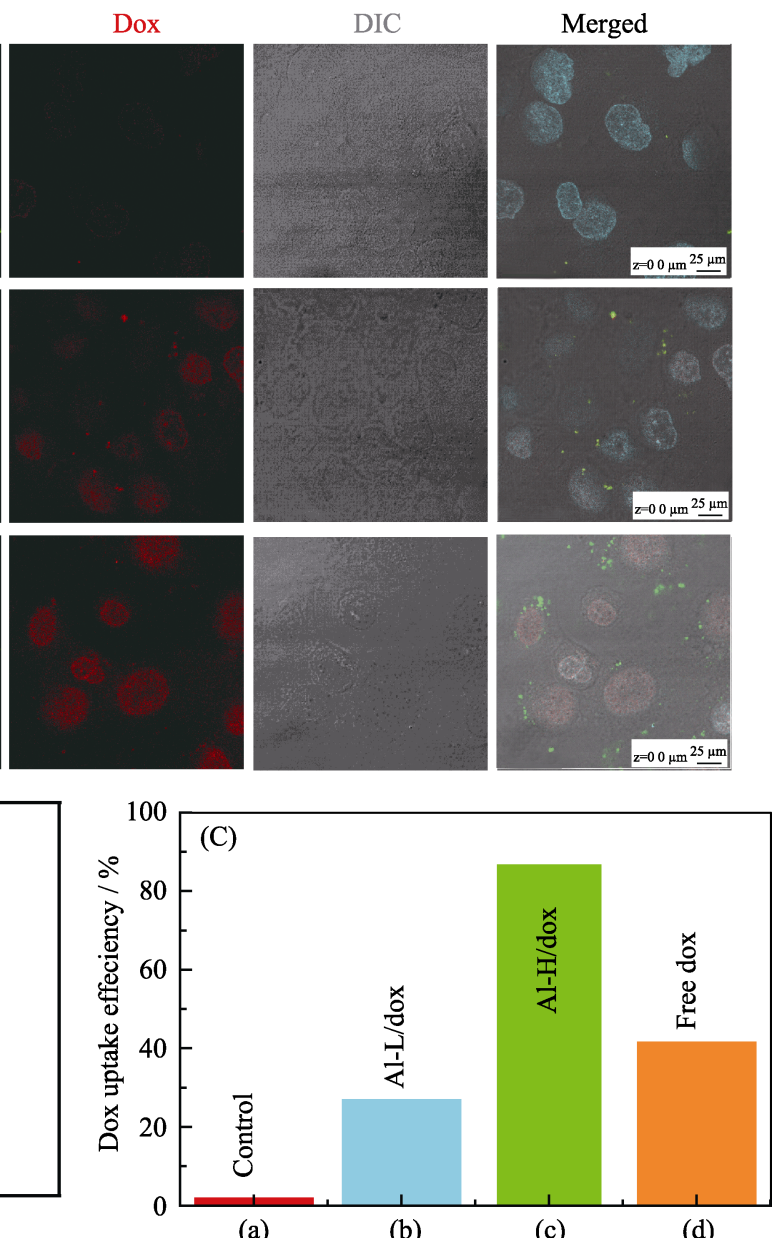

(a)

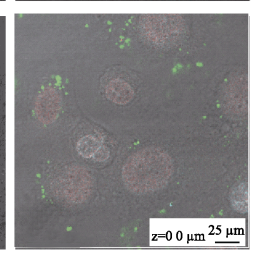

Fig. 2 (A) Confocal laser scanning micrographs of cellular uptake of Al-H/Dox samples by DU145 cells after coculture for 0.5 (a), 2.5 (b) and $24 \mathrm{~h}$ (c); (B) Histogram and (C) quantitative results of Dox cellular uptake efficiency (E\%) by DU145 cells incubated for $2 \mathrm{~h}$ with (a) control, (b) Al-L/Dox at $5 \mu \mathrm{g} / \mathrm{mL}$ of particles, (c) Al-H/Dox at $5 \mu \mathrm{g} / \mathrm{mL}$ of particles, and (d) free Dox with an equivalent amount of Dox loaded onto Al-H/Dox samples
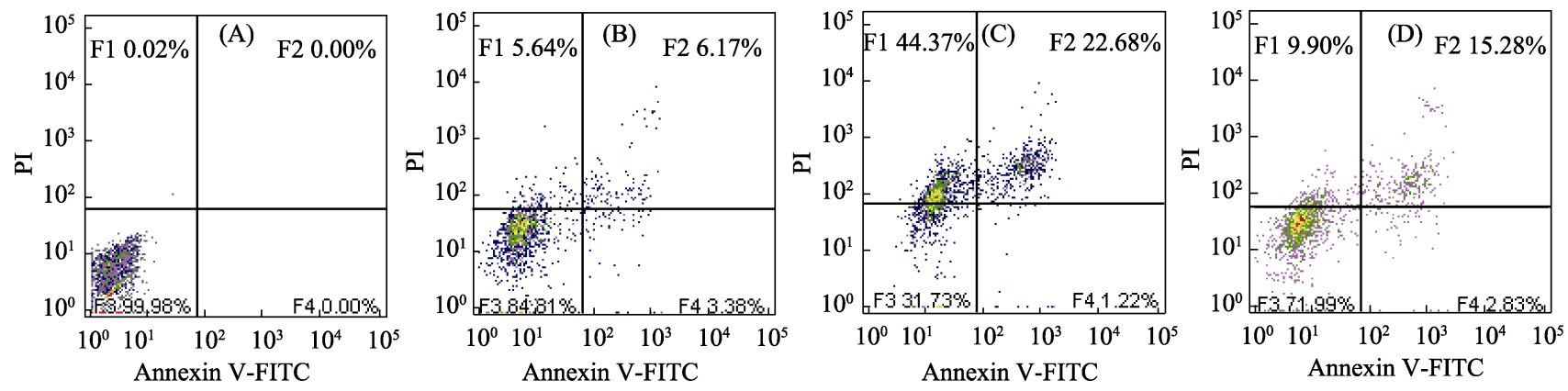

Fig. 3 Percentage of apoptosis and necrosis in DU145 cells treated with control (A), Al-L/Dox at $25 \mu \mathrm{g} / \mathrm{mL}$ (B), or Al-H/Dox at $25 \mu \mathrm{g} / \mathrm{mL}$ (C) for $1 \mathrm{~d}$; Free Dox with an equivalent amount of Dox released from

Al-H/Dox after $1 \mathrm{~d}$ at $\mathrm{pH} 5$ was used as contrast (D)

The ratios of normal, early apoptosis, late apoptosis, and necrosis in DU145 cells treated with Al-L/Dox, Al-H/Dox and free Dox samples (Fig. 3) were studied using double staining with Annexin V-FITC and propidium iodide (PI). The group without any treatment was used as control. Normal live cells were considered to be double negative. Early apoptosis represents cells with Annexin V-FITC positive and PI negative staining. Late apoptosis means double positive cells. Necrosis cells were positive for PI and negative for Annexin V-FITC. Both Al-L/Dox and Al-H/Dox samples showed particleconcentration-dependent cell death. In total, the Al-H/Dox samples displayed substantially higher ratios of cellular death compared with the Al-L/Dox samples. This result is consistent with the Dox loading amount and cellular uptake efficiency. For example, the Al-L/Dox 

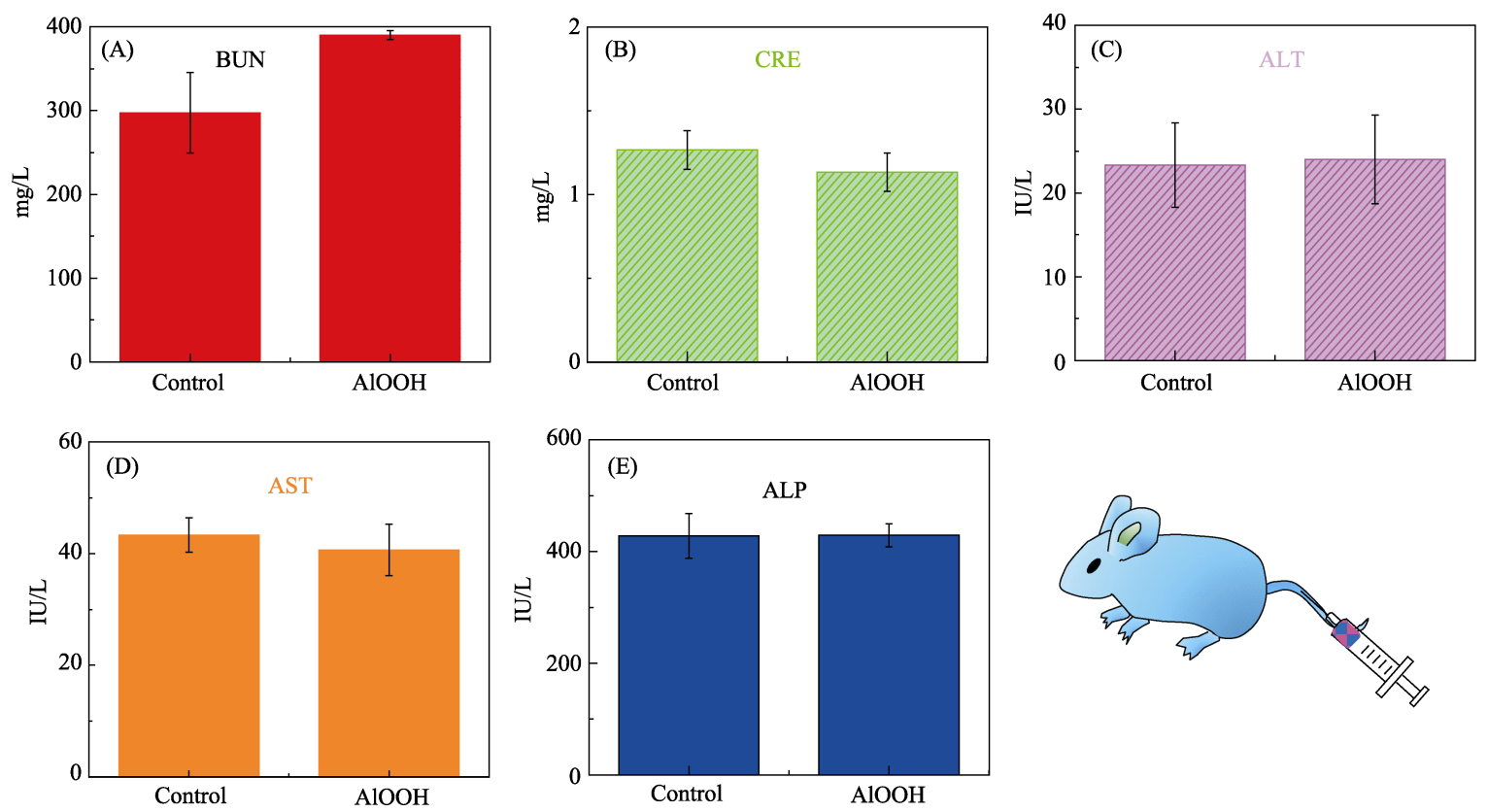

Fig. 4 Blood biochemistry analysis (A-E) of mice after intravenous administration with Al-H $(n=3)$

samples exhibited a ratio of $3.38 \%$ in early apoptosis, $6.17 \%$ in late apoptosis, and $5.64 \%$ in necrosis at $25 \mu \mathrm{g} / \mathrm{mL}$. While, the percentages of late apoptosis and necrosis for the Al-H/Dox samples at $25 \mu \mathrm{g} / \mathrm{mL}$ increased to $22.68 \%$ and $44.37 \%$, respectively, and the percentage of normal cells decreased to $31.73 \%$. In addition, on the basis of the equivalent amount of Dox released from Al-H/Dox after $1 \mathrm{~d}$ at $\mathrm{pH} 5$, the Al-H/Dox samples exhibited higher ratios of late apoptosis and necrosis compared with free Dox.

In vivo toxicology was carried out via intravenous injection of Al-H into C57/BL6 mice (Fig. 4). Then, various kinds of biochemistry parameters, such as blood urea nitrogen (BUN), creatinine (CRE), alanine aminotransferase (ALT), aspartate aminotransferase (AST), and alkaline phosphatase (ALP), were tested. The levels of liver function markers (e.g., ALT, AST, and ALP) and kidney function markers (e.g., $\mathrm{CRE}$ and $\mathrm{BUN}$ ) were within the normal range compared with the control. No obvious hepatic or renal toxicity was observed after the intravenous injection of Al-H.

The AlOOH NSs with a high surface area reduce the side effects of chemotherapy drugs and overcome the influence of MDR. First, the $\mathrm{pH}$-dependent release for Dox-loaded AlOOH NSs is meaningful in decreasing the side effects of chemotherapy drugs, considering that Dox suffers from the dose-dependent side effect of cardiotoxicity $^{[1]}$. Tumor sites are weakly acidic $(\sim 5.8)$ compared with the relatively high $\mathrm{pH}(\sim 7.4)$ in blood or extracellular fluid in normal tissue ${ }^{[21]}$. This condition facilitates the passive targeting of chemotherapy drugs to tumor sites and decreases injury on normal cells based on enhanced permeability and retention effects ${ }^{[4]}$. Moreover, Dox loading onto AlOOH NSs helps overcome MDR, which excludes drug molecules outside the cancer cells owing to the overexpression of certain transporter proteins. The loading of Dox onto AlOOH NSs apparently increased the cellular uptake efficiency of drug molecules compared with that in free form, given that free Dox enters cancer cells through diffusion and Dox-loaded AlOOH NSs are internalized through endocytosis process ${ }^{[22-23]}$. In general, the cellular uptake efficiency through endocytosis is markedly higher than that through diffusion ${ }^{[22-23]}$. The Dox-loaded AlOOH NS particles endocytosed by cancer cells are first located in endosomes with a $\mathrm{pH}$ of around 6 and then fuse with lysosomes at $\mathrm{pH} \sim 5^{[24-25]}$; the condition results in the gradual release of Dox from the carrier.

$\mathrm{AlOOH}$ NSs are promising inorganic carriers with the lowest barrier for clinical transfer. These inorganic components have been used as vaccine adjuvants for humans since the 1920s because of their excellent biocompatibility and safety record as clinically confirmed by medical doctors. In 1926, alum was discovered as an adjuvant in a tetanus toxin vaccine. Although the detailed mechanism for alum as a human vaccine remains controversial and unclear, the synthesis of well-controlled $\mathrm{AlOOH} \mathrm{NSs}$ may facilitate a better understanding of the alum delivery system and broaden its biomedical applications.

\section{Conclusions}

In summary, AlOOH NSs with a tunable pore size and surface area were prepared and used to load chemotherapy drug Dox. AlOOH NSs with high surface area and pore size delivered higher amount of chemotherapy drugs than those with low surface area and pore size. A pH-dependent sustained release behavior was observed for Dox-loaded $\mathrm{AlOOH}$ NSs with high release at a low $\mathrm{pH}$ of around 5 and low release at $\mathrm{pH} \sim 7.4$. The 
Dox-loaded AlOOH NSs exhibited a markedly higher cancer cellular uptake efficiency compared with free-form Dox. Moreover, the Dox-loaded AlOOH NSs with high specific surface area exhibited higher cellular uptake efficiency, apoptosis and necrosis ratios compared with those with a low specific surface area. This study paves the way for the exploration of AlOOH NSs with adjustable nanostructures as promising chemotherapy drug delivery system.

\section{References:}

[1] SINGAL P, LI T M, KUMAR D, et al. Adriamycin-induced heart failure: mechanisms and modulation. Mol. Cell. Biochem., 2000, 207: 77-86.

[2] GILlet J P, GOTTESMAN M M. Mechanisms of multidrug resistance in cancer. Methods. Mol. Biol., 2010, 596: 47-76.

[3] PERSIDIS A. Cancer multidrug resistance. Nat. Biotechnol., 1999, 17: 94-95.

[4] PEER D, KARP J M, HONG S, et al. Nanocarriers as an emerging platform for cancer therapy. Nat. Nanotech., 2007, 2(12): 751-760.

[5] MALTZAHM G V, PARK J H, LIN K Y, et al. Nanoparticles that communicate in vivo to amplify tumour targeting. Nat. Mater., 2011, 10: 545-552.

[6] ASHLEY C E, CARNES E C, PHILliPS G K, et al. The targeted delivery of multicomponent cargos to cancer cells by nanoporous particle-supported lipid bilayers. Nat. Mater., 2011, 10: 389-397.

[7] CHEN Y, TAN C, ZHANG H, et al. Two-dimensional graphene analogues for biomedical applications. Chem. Soc. Rev., 2015, 44: 2681-2701.

[8] CHIMENE D, ALGE D L, GAHARWAR A K. Two-dimensional nanomaterials for biomedical applications: emerging trends and future prospects. Adv. Mater., 2015, 27: 7261-7284.

[9] WU J, ZHU Y J, CHEN F. Ultrathin calcium silicate hydrate nanosheets with large specific surface areas: synthesis, crystallization, layered self-assembly and applications as excellent adsorbents for drug, protein, and metal ions. Small, 2013, 9: 2911-2925.

[10] EISENBARTH S C, COLEGIO O R, JR W O C, et al. Crucial role for the Nalp3 inflammasome in the immunostimulatory properties of aluminium adjuvants. Nature, 2008, 453(7198): 1122-1126.

[11] FLACH T L, NG G, HARI A, et al. Alum interaction with dendritic cell membrane lipids is essential for its adjuvanticity. Nat. Med., 2011, 17: 479-487.

[12] MARICHAL T, OHATA K, BEDORET D, et al. DNA released from dying host cells mediates aluminum adjuvant activity. Nat. Med., 2011, 17: 996-1002.

[13] LI X, WANG X P, ITO A. Tailoring inorganic nanoadjuvants towards next-generation vaccines. Chem. Soc. Rev., 2018, 47: 4954- 4980.

[14] SUN B, JI Z, LIAO Y P, et al. Engineering an effective immune adjuvant by designed control of shape and crystallinity of aluminum oxyhydroxide nanoparticles. ACS Nano., 2013, 7: 10834-10849.

[15] CAI W, YU J, MANN S. Template-free hydrothermal fabrication of hierarchically organized $\gamma$-AlOOH hollow microspheres. Micro. Meso. Mater., 2009, 122: 42-47.

[16] LI G, GUAN L, LIU Y, et al. Template-free solvothermal synthesis of 3D hierarchical nanostructured boehmite assembled by nanosheets. J. Phys. Chem. Solids., 2012, 73: 1055-1060.

[17] WANG X P, LI X, SOGO Y, et al. Simple synthesis route of mesoporous $\mathrm{AlOOH}$ nanofibers to enhance immune responses. RSC. Adv., 2013, 3: 8164-8167.

[18] WEN J R, LIU M H, MOU C Y. Synthesis of curtain-like crumpled boehmite and $\gamma$-alumina nanosheets. CrystEngComm., 2015, 17: 1959-1967.

[19] HICKS R W, PINNAVAIA T J. Nanoparticle assembly of mesoporous AlOOH (Boehmite). Chem. Mater., 2003, 15: 78-82.

[20] LI X, WANG X, HUA Z, et al. One-pot synthesis of magnetic and mesoporous bioactive glass composites and their sustained drug release property. Acta. Mater., 2008, 56: 3260-3265.

[21] BARRETO J A, O'MALLEY W, KUBEIL M, et al. Nanomaterials: applications in cancer imaging and therapy. Adv. Mater., 2011, 23: H18-H40.

[22] BILDSTEIN L, DUBERNET C, COUVREUR P. Prodrug-based intracellular delivery of anticancer agents. Adv. Drug. Delivery. Rev., 2011, 63: 3-23.

[23] SKOVSGAARD T, NISSEN N I. Membrane transport of anthracyclines. Pharmacol. Therapeut., 1982, 18: 293-311.

[24] LI X, WANG X, SOGO Y, et al. Mesoporous silica-calcium phosphatetuberculin purified protein derivative composites as an effective adjuvant for cancer immunotherapy. Adv. Healthcare. Mater., 2013, 2: 863-871.

[25] BLOEBAUM R D, LUNDEEN G A, BACHUS K N, et al. Dissolution of particulate hydroxyapaptite in a macrophage organelle model. J. Biomed. Mater. Res., 1998, 40: 104-114.

\section{氢氧化铝纳米片: 结构依赖性癌症化疗药物的储运}

\section{Xia ${ }^{1}$, SHENASHEN Mohamed A ${ }^{1}$, MEKAWY Moataz ${ }^{1}$, TANIGUCHI Akiyoshi ${ }^{2,3}$, EI-SAFTY Sherif $A^{1,3}$}

(1. Research Center for Functional Materials, National Institute for Materials Science, Ibaraki 305-0047, Japan; 2. Cellular Functional Nanomaterials Group, Research Center for Functional Materials, National Institute for Materials Science, Tsukuba, Ibaraki 305-0044, Japan; 3. Engineering and Advanced Manufacturing, University of Sunderland, Sunderland, United Kingdom)

摘 要: 铝盐佐剂具有极好的安全记录, 是各种人类疫苗中唯一获得 FDA 许可的无机佐剂。据我们所知, 目前尚没 有关于将其用作化疗药物的递送系统、并系统阐明其结构与载药性能之间关系的研究报道。本研究采用三嵌段共 聚物、通过调节反应时间合成了具有可调比表面积和孔径的氢氧化铝 $(\mathrm{AlOOH})$ 纳米片。AlOOH 纳米片的最大比表 面积达 $470 \mathrm{~m}^{2} / \mathrm{g}$ 。其负载化疗药物阿需素的能力与材料结构密切相关: 比表面积和孔径越大, 负载化疗药物的量越 大。负载有阿霉素的 $\mathrm{AlOOH}$ 纳米片呈现与 $\mathrm{pH}$ 有关的药物释放行为: 在 $\mathrm{pH} 5$ 的低 $\mathrm{pH}$ 环境下快速释放, 而在 $\mathrm{pH} 7.4$ 的近中性 $\mathrm{pH}$ 下缓慢释放。流式细胞术显示, 相比于游离形式的阿霉素, 负载在 $\mathrm{AlOOH}$ 纳米片上的阿霉素更易被 癌细胞所吞噬。而且负载阿霉素后, 与低比表面积的 $\mathrm{AlOOH}$ 纳米片相比, 高比表面积的 $\mathrm{AlOOH}$ 纳米片更有利于 被癌细胞摄取、诱导癌细胞调亡和坏死。因此, 本研究所合成的 $\mathrm{AlOOH}$ 纳米片有望用作化疗药物递送体系。

关 键 词: 氢氧化铝; 纳米片; 癌症化疗; 存储; 药物输送

中图分类号:TQ174 文献标识码: A 